\title{
Impurity Effect on the In-plane Penetration Depth of the Organic Superconductors $\kappa-(\mathrm{BEDT}-\mathrm{TTF})_{2} X\left(X=\mathrm{Cu}(\mathrm{NCS})_{2}\right.$ and $\left.\mathrm{Cu}\left[\mathrm{N}(\mathrm{CN})_{2}\right] \mathrm{Br}\right)$
}

\author{
N. Yoneyama, A. Higashihara, T. Sasaki \\ Institute for Materials Research, Tohoku University, Sendai 980-8577, Japan \\ T. Nojima \\ Center for Low Temperature Science, Tohoku University, Sendai 980-8577, Japan \\ N. Kobayashi \\ Institute for Materials Research, Tohoku University, Sendai 980-8577, Japan \\ Center for Low Temperature Science, Tohoku University, Sendai 980-8577, Japan
}

(Dated: October 30, 2018)

\begin{abstract}
We report the in-plane penetration depth $\lambda_{\|}$of single crystals $\kappa$-(BEDT-TTF $)_{2} X\left(X=\mathrm{Cu}(\mathrm{NCS})_{2}\right.$ and $\left.\mathrm{Cu}\left[\mathrm{N}(\mathrm{CN})_{2}\right] \mathrm{Br}\right)$ by means of the reversible magnetization measurements under the control of cooling-rate. In $X=\mathrm{Cu}(\mathrm{NCS})_{2}, \lambda_{\|}(0)$ as an extrapolation toward $T=0 \mathrm{~K}$ does not change by the cooling-rate within the experimental accuracy, while $T_{\mathrm{c}}$ is slightly reduced. On the other hand, in $X=\mathrm{Cu}\left[\mathrm{N}(\mathrm{CN})_{2}\right] \mathrm{Br}, \lambda_{\|}(0)$ indicates a distinct increase by cooling faster. The different behavior of $\lambda_{\|}(0)$ on cooling-rate between the two salts is quantitatively explained in terms of the local-clean approximation (London model), considering that the former salt belongs to the very clean system and the later the moderate clean one. The good agreement with this model demonstrates that disorders of ethylene-group in BEDT-TTF introduced by cooling faster increase the electron(quasiparticle)scattering, resulting in shorter mean free path.
\end{abstract}

\section{INTRODUCTION}

The organic superconductors $\kappa$-(BEDT-TTF $)_{2} X(X$ $=\mathrm{Cu}(\mathrm{NCS})_{2}$ and $\left.\mathrm{Cu}\left[\mathrm{N}(\mathrm{CN})_{2}\right] \mathrm{Br}\right)$ belong to a family of charge transfer salts, where BEDT-TTF is bis(ethylenedithio)-tetrathiafulvalene. The crystal structure consists of alternating layers of conducting BEDTTTF and insulating $X$ anions $\frac{1}{-1}$ Consequently, these systems are characterized by highly anisotropic properties. High purity in samples makes it possible to observe

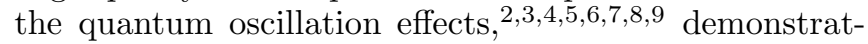
ing the quasi-two-dimensional electronic states. In the superconducting state, these salts are described in terms of type-II superconductor with long in-plane penetration depth $\lambda_{\|}$and short in-plane coherence length $\xi_{\|}$as described below.

The in-plane penetration depth is one of the fundamental parameters of superconductivity, because the temperature-dependence of $\lambda_{\|}$at low temperatures is reflected by gap excitation in the superconducting state, giving information on the gap symmetry, i.e., full-gap or node-gap, etc. Thus there have been a large number of reports on $\lambda_{\|}$as a function of

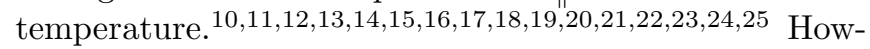
ever, prior to such a delicate discussion, it should be noticed that the wide distribution of the absolute values of $\lambda_{\|}(0)$ has been reported, taking into account the following inconsistency in $\lambda_{\|}(0)$ between experimental methods. The values of $\lambda_{\|}(0)$ in the

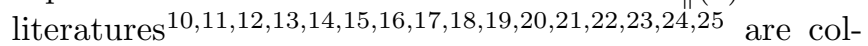
lected in Tables【and! All the measurements have been carried out at the same configuration of magnetic-field $H$ perpendicular to the conduction plane. Nevertheless one can find that they are classified into two groups; one is with $\lambda_{\|}(0)$ longer than $\sim 1 \mu \mathrm{m}$ (surface impedance,,$\stackrel{10.11}{ }$ ac inductance,,$\frac{12}{1}$ and ac susceptibility ${ }^{20,21}$ ) and the other with shorter $\lambda_{\|}(0)(\mu \mathrm{SR}, 15.16 .17$ magnetization,, $19.24 .25$ and decoration $\left.\operatorname{method}^{18}\right)$. The former measurements are performed in magnetic-field lower than $H_{\mathrm{c} 1}$. In this situation, shielding current flows around the sample surface to exclude $H$ (shielding states), and then $\lambda_{\|}$is obtained as the penetrating length of $H$ at the sample edge (Fig. 1(a)). On the other hand, the latter measurements are carried out in magnetic-field much larger than $H_{\mathrm{c} 1}$, where $H$ penetrates into the sample as vortices (mixed states). In this case, $\lambda_{\|}$is given as a decay length of the magnetic-field from the center of the vortex (Fig. [(b)). As shown in Tables \ and III the former group seems to overestimate $\lambda_{\|}(0)$ compared to the latter one, because the former methods may be sensitive to the surface states. Up to now, only little effort has been spent on this controversial point.

In the superconducting state of $X=\mathrm{Cu}(\mathrm{NCS})_{2}$, hereafter abbreviated as the $\mathrm{Cu}(\mathrm{NCS})_{2}$ salt, the local and clean limit is adequate to the systems; $\lambda_{\|}>$ $l_{\|}>\xi_{\|}$, where the intermediate in-plane mean free path $l_{\|}=100-240 \mathrm{~nm}^{2.3 .4 .5 .6 .26}$ and the short $\xi_{\|}=$ $3.1-7 \mathrm{~nm} 11.16 .27$ Compared with this salt, $l_{\|}$of $X=$ $\mathrm{Cu}\left[\mathrm{N}(\mathrm{CN})_{2}\right] \mathrm{Br}$ (the $\mathrm{Cu}\left[\mathrm{N}(\mathrm{CN})_{2}\right] \mathrm{Br}$ salt) is remarkably short: $l_{\|}=26-38 \mathrm{~nm}^{7.8 .9}$, while $\xi_{\|}\left(=2.4-3.7 \mathrm{~nm}^{11.27}\right)$ is almost comparable to the $\mathrm{Cu}(\mathrm{NCS})_{2}$ salt. On the basis of such the local-clean approximation (London model) ${ }^{28}$ $\lambda_{\|}(0)$ is described as

$$
\lambda_{\|}(0)=\lambda_{\mathrm{L}}(0)\left(1+\xi_{0} / l_{\|}\right)^{0.5},
$$

where $\lambda_{\mathrm{L}}(0)$ is the London penetration depth for a pure 
sample and $\xi_{0}$ is the coherence length. In eq. (1), when $l_{\|}$ becomes short with increasing impurity scattering, $\lambda_{\|}(0)$ becomes longer than $\lambda_{\mathrm{L}}(0)$.

A possible impurity of the present materials is originated from a feature of molecular-based compounds, because an internal degree of freedom in the donor molecule can give a positional disorder. At room temperature, conformation of the terminal ethylene groups in BEDT-TTF is thermally excited. ${ }^{29}$ Such conformational disorders (ethylene-disorders) are frozen by cooling very fast (quenching) and may affect the scattering of carriers at low temperatures. Especially in the $\mathrm{Cu}\left[\mathrm{N}(\mathrm{CN})_{2}\right] \mathrm{Br}$ salt, as mentioned above, $l_{\|}$is shorter than that of the $\mathrm{Cu}(\mathrm{NCS})_{2}$ salt, and so the electronic system of the $\mathrm{Cu}\left[\mathrm{N}(\mathrm{CN})_{2}\right] \mathrm{Br}$ salt will be slightly dirty. In addition, several investigations have been reported in the $\mathrm{Cu}\left[\mathrm{N}(\mathrm{CN})_{2}\right] \mathrm{Br}$ salt under the control of cooling-rate. The residual resistivity increases by cooling faster and simultaneously the superconducting transition temperature $T_{\mathrm{c}}$ decreases. ${ }^{30}$ In a Shubnikov-de Haas (SdH) oscillation measurement,$\frac{9}{9}$ the oscillation amplitude is suppressed with increasing disorders. These results imply that the ethylene-disorders play an important role on the electron (or quasiparticle)-scattering as an impurity origin.

Aburto et al..$^{25}$ have reported a slight increase of $\lambda_{\|}(0)$ by cooling fast (a magnetization measurement in the mixed state). On the other hand, an ac susceptibility measurement ${ }^{21}$ (in the shielding state) provides an extremely large increase of $\lambda_{\|}(0)$ by quenching, up to $\sim 100 \mu \mathrm{m}$. Motivated by this inconsistency in the behavior of $\lambda_{\|}(0)$ between the experimental methods, we present in this paper a quantitative explanation for the increase of $\lambda_{\|}(0)$ by the impurity effect originated from ethylene-disorders.

We evaluate $\lambda_{\|}(0)$ from dc reversible magnetization measurements in the mixed state under the control of cooling-rate. This method gives a reliable estimate of the penetration depth in bulk samples, because a homogeneous magnetic-field penetration appears around vortices free from pinning. This investigation reveals that a distinct difference appears between the two salts on the behavior of the cooling-rate dependence of $\lambda_{\|}(0)$. In the $\mathrm{Cu}(\mathrm{NCS})_{2}$ salt, $\lambda_{\|}(0)$ is not changed by quenching within the experimental accuracy, while $T_{\mathrm{c}}$ decreases slightly. In contrast, in the $\mathrm{Cu}\left[\mathrm{N}(\mathrm{CN})_{2}\right] \mathrm{Br}$ salt, a significant change in $\lambda_{\|}(0)$ is observed with increasing cooling-rate. We demonstrate that this behavior is well described using the local-clean limit approximation in eq. (11). The ethylene-disorders introduced by cooling faster will increase the electron (quasiparticle)-scattering, resulting in shorter mean free path. A preliminary report is given in Ref. 31.
TABLE I: A list of in-plane penetration depths in $\kappa$-(BEDT$\mathrm{TTF})_{2} \mathrm{Cu}(\mathrm{NCS})_{2}$.

\begin{tabular}{cccc}
\hline magnetic-field & experiments & $\lambda_{\|}(0)(\mu \mathrm{m})$ & Ref. \\
\hline$H<H_{\mathrm{c} 1}$ & surface impedance & 2 & 10 \\
& surface impedance & 1.4 & 11 \\
& ac inductance & 1.8 & 12 \\
& ac susceptibility & - & 13.14 \\
\cline { 2 - 4 }$H>H_{\mathrm{c} 1}$ & $\mu \mathrm{SR}$ & 0.98 & 15 \\
& $\mu \mathrm{SR}$ & 0.768 & 16 \\
& $\mu \mathrm{SR}$ & 0.43 & 17 \\
& decoration & 0.4 & 18 \\
& magnetization & 0.535 & 19 \\
& magnetization & 0.43 & this work \\
\hline
\end{tabular}

TABLE II: A list of in-plane penetration depths in $\kappa$-(BEDT$\mathrm{TTF})_{2} \mathrm{Cu}\left[\mathrm{N}(\mathrm{CN})_{2}\right] \mathrm{Br}$.

\begin{tabular}{cccc}
\hline magnetic-field & experiments & $\lambda_{\|}(0)(\mu \mathrm{m})$ & Ref. \\
\hline$H<H_{\mathrm{c} 1}$ & surface impedance & 1.5 & 11 \\
& ac inductance & 3.2 & 12 \\
& ac susceptibility & $\sim 1$ & 20 \\
& ac susceptibility & $1.5(\mathrm{R})-100(\mathrm{Q})^{a}$ & 21 \\
& ac susceptibility & $1.1(\mathrm{~A})-24(\mathrm{Q})^{b}$ & 21 \\
\cline { 2 - 4 }$H>H_{\mathrm{c} 1}$ & $\mu$ SR & - & 22 \\
& decoration & 0.65 & 23 \\
& magnetization & 24 \\
& magnetization & $0.58(\mathrm{~S})-0.64(\mathrm{R})^{c}$ & 25 \\
& magnetization & $0.57(\mathrm{~S})-0.69(\mathrm{Q})^{d}$ this work \\
\hline
\end{tabular}

${ }^{a} \mathrm{R}: \sim 0.2 \mathrm{~K} / \mathrm{min}, \mathrm{Q}: \sim 300 \mathrm{~K} / \mathrm{min}$.

${ }^{b} \mathrm{~A}$ : annealed at $78-100 \mathrm{~K}, \mathrm{Q}: \sim 300 \mathrm{~K} / \mathrm{min}$.

${ }^{c} \mathrm{~S}: 1.4 \mathrm{~K} / \mathrm{min}, \mathrm{R}: 20 \mathrm{~K} / \mathrm{min}$.

${ }^{d}$ S: slow-cooled, Q: quenched.

\section{EXPERIMENTAL}

Single crystals were grown by a standard electrochemical technique. The dimensions of samples were $2.2 \times 1.1 \times$ $0.40 \mathrm{~cm}^{3}$ (the $\mathrm{Cu}(\mathrm{NCS})_{2}$ salt) and $1.1 \times 1.0 \times 0.13 \mathrm{~mm}^{3}$ $\left(\mathrm{Cu}\left[\mathrm{N}(\mathrm{CN})_{2}\right] \mathrm{Br}\right)$. The samples were cooled with a rate of $\sim 100 \mathrm{~K} / \mathrm{min}$ from room temperature to $15 \mathrm{~K}$, making "quenched" state. Following the measurements in the quenched state, two cooling procedures were carried out in series with $15 \mathrm{~K} / \mathrm{min}$ (rapid-cooled) and $0.2 \mathrm{~K} / \mathrm{min}$ (slow-cooled) in each cooling process after warmed up to $100 \mathrm{~K}$. In the different cooling states, a series of the mag-
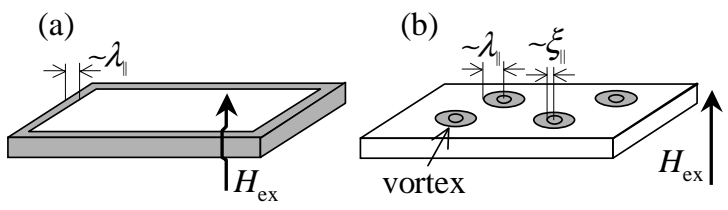

FIG. 1: Schematic view of the in-plane penetration depth in (a) the shielding state and (b) the mixed state. Bold arrows indicate the magnetic-field perpendicular to the conduction plane. The gray-shaded regions indicate the penetrating area of the magnetic-field. 


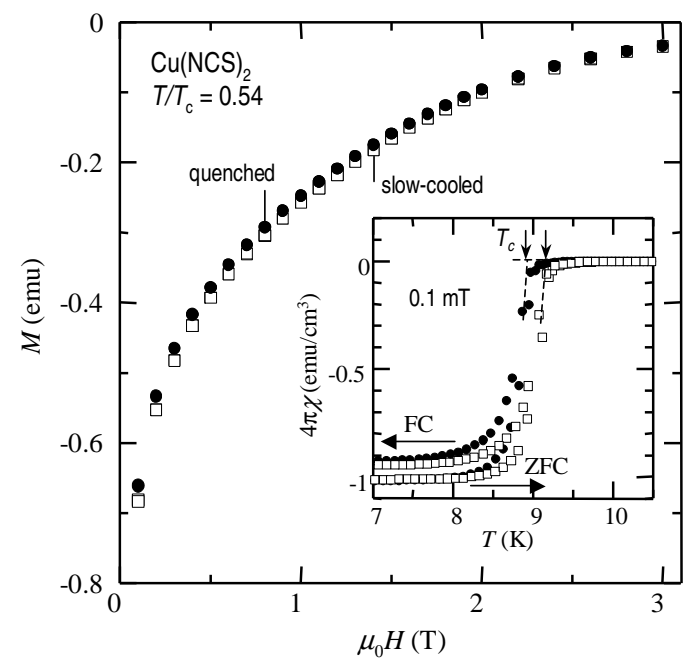

FIG. 2: Magnetization curves of $\kappa$-(BEDT-TTF $)_{2} \mathrm{Cu}(\mathrm{NCS})_{2}$ at $T / T_{\mathrm{c}}=0.54$ for the slow-cooled (open squares, $T / T_{\mathrm{c}}=$ $5.0 \mathrm{~K} / 9.2 \mathrm{~K}$ ) and quenched states (filled circles, $T / T_{\mathrm{c}}=4.8$ $\mathrm{K} / 8.9 \mathrm{~K})$. The inset shows the temperature dependence of the magnetic susceptibilities at $0.1 \mathrm{mT}$ under zero-field-cooling (ZFC) and field-cooling (FC) conditions.

netization measurements was performed using a SQUID magnetometer (Quantum Design, MPMS-5 and MPMS7). The magnetic-field was applied perpendicular to the conduction plane. The similar experiments for other crystals indicated good reproducibility with the data presented here for both salts.

For type-II superconductor in the magnetic-field regime $\left(H_{\mathrm{c} 1}<\right) H_{\mathrm{irr}}<H \ll H_{\mathrm{c} 2}$, the London mode ${ }^{32.33}$ describes the magnetization $M$ as a function of $H$ as

$$
-4 \pi M=\left(\alpha \phi_{0} / 8 \pi \lambda_{\|}^{2}\right) \ln \left(H_{\mathrm{c} 2} \beta / H\right)
$$

where $H_{\text {irr }}$ is the irreversibility field of the magnetizations, $\phi_{0}$ the magnetic flux quantum, and $\beta$ a constant of order unity. While a correction factor $\alpha$ is unity for the conventional London model, Hao and $\mathrm{Clem}^{33}$ have corrected the vortex-core contribution neglected in the London model, which provided $\alpha=0.70$ in the present magnetic-field range. According to eq. (2), $\lambda_{\|}$was estimated from the slope of the linear dependence in the $M$ vs $\ln H$ plot, where the slope is $\alpha \phi_{0} / 32 \pi^{2} \lambda_{\|}^{2}$.

\section{RESULTS}

The insets of Figs. 2 and 3 show the magnetic susceptibility of $X=\mathrm{Cu}(\mathrm{NCS})_{2}$ and $\mathrm{Cu}\left[\mathrm{N}(\mathrm{CN})_{2}\right] \mathrm{Br}$ at $0.1 \mathrm{mT}$, respectively. After subtracting the contribution of the core diamagnetization, the demagnetization factor was corrected using an ellipsoidal approximation. In both salts, $T_{\mathrm{c}}$ decreases with increasing cooling-rate: $9.2 \mathrm{~K}$ (slow-cooled) and $8.9 \mathrm{~K}$ (quenched) for the $\mathrm{Cu}(\mathrm{NCS})_{2}$ salt, $11.7 \mathrm{~K}$ (slow-cooled), $11.4 \mathrm{~K}$ (rapid-cooled), and

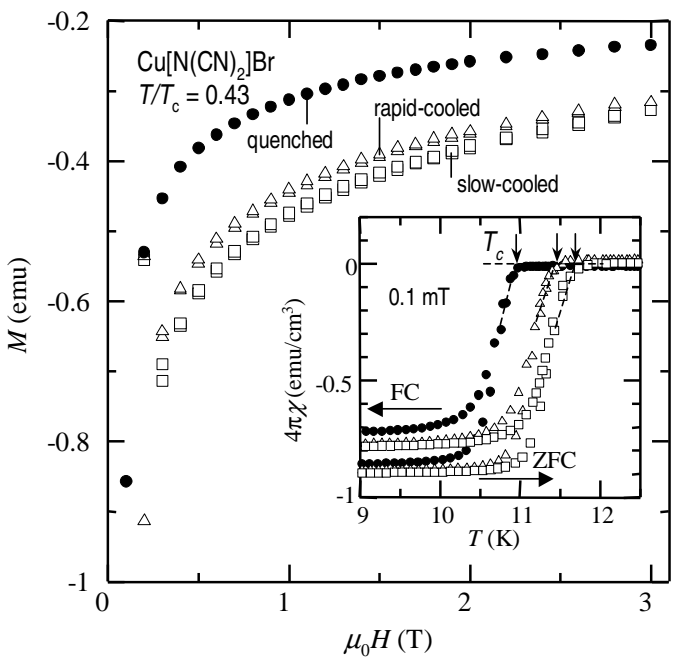

FIG. 3: Magnetization curves of $\kappa$-(BEDT$\mathrm{TTF})_{2} \mathrm{Cu}\left[\mathrm{N}(\mathrm{CN})_{2}\right] \mathrm{Br}$ at $T / T_{\mathrm{c}}=0.43$ for the slow-cooled (open squares, $T / T_{\mathrm{c}}=5.0 \mathrm{~K} / 11.7 \mathrm{~K}$ ), rapid-cooled (open triangles, $\left.T / T_{\mathrm{c}}=4.9 \mathrm{~K} / 11.4 \mathrm{~K}\right)$, and quenched states (filled circles, $T / T_{\mathrm{c}}=4.7 \mathrm{~K} / 10.9 \mathrm{~K}$ ). The inset shows the temperature dependence of the magnetic susceptibilities at $0.1 \mathrm{mT}$ under zero-field-cooling (ZFC) and field-cooling (FC) conditions.

$10.9 \mathrm{~K}$ (quenched) for the $\mathrm{Cu}\left[\mathrm{N}(\mathrm{CN})_{2}\right] \mathrm{Br}$ salt. Here $T_{\mathrm{c}}$ 's are defined as an intercept of the extrapolated lines of the normal and superconducting states (broken lines and arrows in the insets of Figs. 2 and 31). The cooling-rate dependence of $T_{\mathrm{c}}$ for the $\mathrm{Cu}\left[\mathrm{N}(\mathrm{CN})_{2}\right] \mathrm{Br}$ salt is consistent with the literatures 21.25 .30 .34 .35 .36 as shown in Fig. 4. This plot indicates that $T_{\mathrm{c}}$ changes with almost an unified slope as a function of cooling-rate $s(\mathrm{~K} / \mathrm{min})$ : $\left|\Delta T_{\mathrm{c}} / \Delta \log (s)\right|=0.2-0.3$, whereas the absolute values of $T_{\mathrm{c}}$ different between the reports may come from the different definition of $T_{\mathrm{c}}$. The magnitude of the reduction in $T_{\mathrm{c}}$ for the $\mathrm{Cu}(\mathrm{NCS})_{2}$ salt is much smaller than that for the $\mathrm{Cu}\left[\mathrm{N}(\mathrm{CN})_{2}\right] \mathrm{Br}$ salt. The susceptibilities under a zero-field-cooled condition (ZFC) indicate almost the full Meissner volume. The negligible cooling-rate dependence of the superconducting volume is consistent with the reported results, $\stackrel{35.36}{5}$ whereas Pinterić et al. have reported a small suppression of the volume by cooling faster ${ }^{21}$

The main panels of Figs. 2 and 3 show the magnetization curves at $T / T_{\mathrm{c}}=0.54$ (the $\mathrm{Cu}(\mathrm{NCS})_{2}$ salt) and 0.43 (the $\mathrm{Cu}\left[\mathrm{N}(\mathrm{CN})_{2}\right] \mathrm{Br}$ salt). The data at $5.0 \mathrm{~K}$ are shown for both slow-cooled states, and the corresponding data with the same $T / T_{\mathrm{c}}$ value for the rapid-cooled and quenched states are estimated from linear interpolations with the data at 4.5 and $5.0 \mathrm{~K}$. The irreversibility field around $5 \mathrm{~K}$ is about $80 \mathrm{mT}$ for the $\mathrm{Cu}(\mathrm{NCS})_{2}$ salt and about $200-300 \mathrm{mT}$ for the $\mathrm{Cu}\left[\mathrm{N}(\mathrm{CN})_{2}\right] \mathrm{Br}$ salt. Within the experimental accuracy, no change of the reversible $M$ by quenching is observed in the $\mathrm{Cu}(\mathrm{NCS})_{2}$ salt. On the contrary, in the $\mathrm{Cu}\left[\mathrm{N}(\mathrm{CN})_{2}\right] \mathrm{Br}$ salt, a large difference in $M$ appears by changing cooling-rate. 


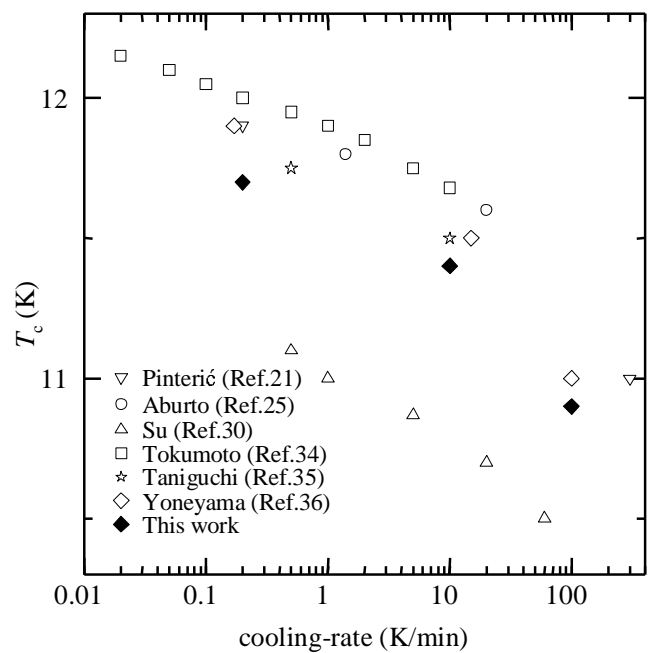

FIG. 4: Cooling-rate dependence of $T_{\mathrm{c}}$ in $\kappa$-(BEDT$\mathrm{TTF})_{2} \mathrm{Cu}\left[\mathrm{N}(\mathrm{CN})_{2}\right] \mathrm{Br}$. The data of the present work are displayed with filled diamonds. Open symbols are the data taken from the literatures. ${ }^{21.25 .30 .34 .35 .36}$

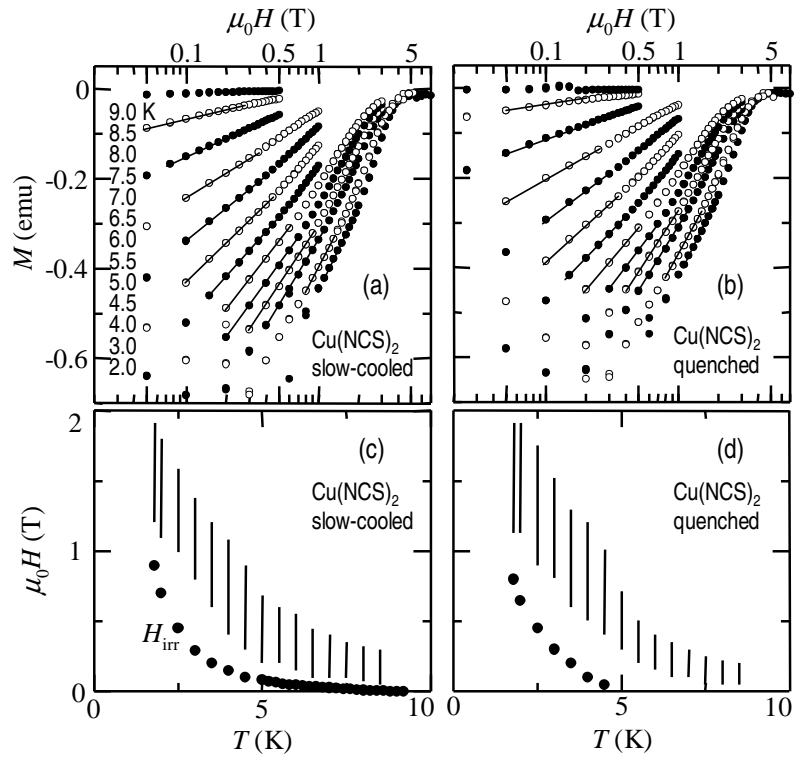

FIG. 5: Magnetization vs $\ln H$ plots in $\kappa$-(BEDT$\mathrm{TTF})_{2} \mathrm{Cu}(\mathrm{NCS})_{2}$ for (a) the slow-cooled and (b) quenched states. Lower panels (c) and (d) indicate the irreversibility field $H_{\text {irr }}$ (filled circles) and the region for the linear reversible magnetization in $\ln H$ (solid vertical bars) for each coolingrate.

The reversible magnetizations are depicted on a semilogarithmic scale in Figs. 5(a), 5(b), 6(a), and 6(b). In this plot a linear-dependent region emerges (solid lines), except for the data of the $\mathrm{Cu}\left[\mathrm{N}(\mathrm{CN})_{2}\right] \mathrm{Br}$ salt below 3.0 K (not shown in the Figures). The linear regions are also displayed in Figs. 5] (c), 5(d), 6(c), and 6](d) as solid vertical bars, which satisfy the condition

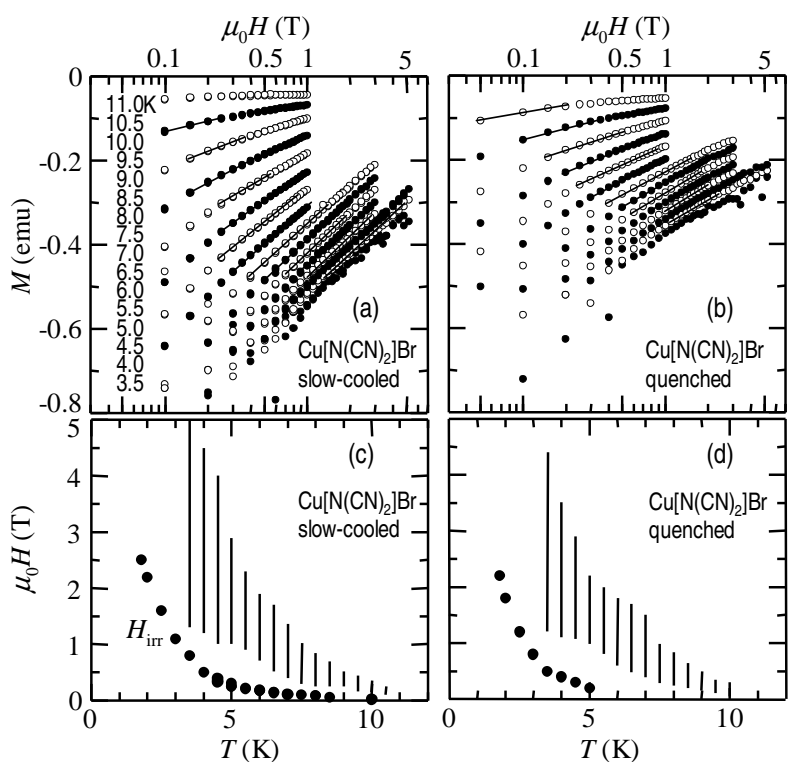

FIG. 6: Magnetization vs $\ln H$ plots in $\kappa$-(BEDT$\mathrm{TTF})_{2} \mathrm{Cu}\left[\mathrm{N}(\mathrm{CN})_{2}\right] \mathrm{Br}$ for (a) the slow-cooled and (b) quenched states. Lower panels (c) and (d) indicate the irreversibility field $H_{\text {irr }}$ (filled circles) and the region for the linear reversible magnetization in $\ln H$ (solid vertical bars) for each cooling-rate.

$H_{\text {irr }}<H \ll H_{\mathrm{c} 2}$ and thus guarantee the appropriate determination of the penetration depth.

From these slopes, the penetration depths are obtained as shown in Fig. [7] In the $\mathrm{Cu}(\mathrm{NCS})_{2}$ salt, we do not find any difference in $\lambda_{\|}(T)$ at low temperatures between the two cooling-rates within the accuracy of the measurement. In order to estimate $\lambda_{\|}(0)$, we adopt a linear extrapolation to $T=0 \mathrm{~K}$, giving $\lambda_{\|}(0)=430 \pm 20 \mathrm{~nm}$ (slow-cooled and quenched). Here, it should be noted that it is difficult to obtain meaningful fits to the present data as a function of $T$, and thus the $T$-linear dependence employed here is not worth discussing in detail.

In contrast to the $\mathrm{Cu}(\mathrm{NCS})_{2}$ salt, a distinct increase of $\lambda_{\|}$by cooling faster is observed in the $\mathrm{Cu}\left[\mathrm{N}(\mathrm{CN})_{2}\right] \mathrm{Br}$ salt. Similar estimates of $\lambda_{\|}(0)$ by linear extrapolations result in $570 \pm 30 \mathrm{~nm}$ (slow-cooled), $590 \pm 30 \mathrm{~nm}$ (rapid-cooled), and $690 \pm 50 \mathrm{~nm}$ (quenched). This change of $\lambda_{\|}(0)$ can be also seen in the original $M(\ln H)$ curves shown in Fig. 6. where the slope in the slow-cooled state (Fig. 6(a)) is suppressed by quenching (Fig. 6(b)), while the corresponding data for the $\mathrm{Cu}(\mathrm{NCS})_{2}$ salt do not show such a cooling-rate dependence of the slope (Figs. 5(a,b)).

\section{DISCUSSION}

First we compare the absolute values of $\lambda_{\|}(0)$ in the slow-cooled state with the reported results summarized in Tables II and II] $\lambda_{\|}(0)$ obtained in the present work is $430 \pm 20$ and $570 \pm 30 \mathrm{~nm}$ for the 


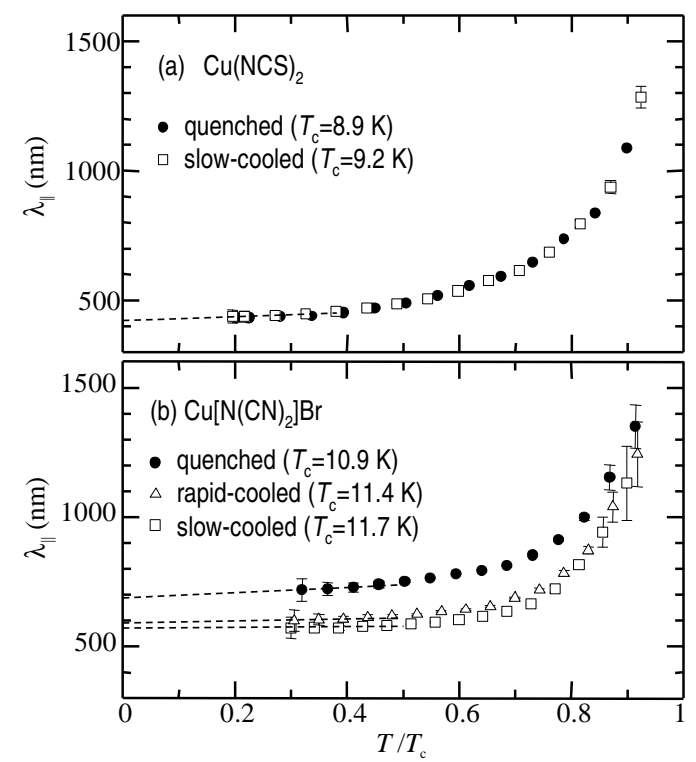

FIG. 7: Temperature dependence of the in-plane penetration depth for $\kappa$-(BEDT-TTF $)_{2} X$, where $X=\left(\right.$ a) $\mathrm{Cu}(\mathrm{NCS})_{2}$ and (b) $\mathrm{Cu}\left[\mathrm{N}(\mathrm{CN})_{2}\right] \mathrm{Br}$. Broken lines are linear extrapolations toward $T=0 \mathrm{~K}$, providing $\lambda_{\|}(0)$.

$\mathrm{Cu}(\mathrm{NCS})_{2}$ and $\mathrm{Cu}\left[\mathrm{N}(\mathrm{CN})_{2}\right] \mathrm{Br}$ salts, respectively. The values of $\lambda_{\|}(0)$ are almost comparable to the data by magnetization, ${ }^{19.24 .25} \mu \mathrm{SR}, \stackrel{15.16 .17}{{ }^{10}}$ and decoration ${ }^{18}$ measurements, which have been carried out in the mixed states. The other reports performed in the shielding

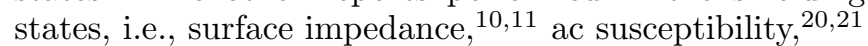
and ac inductance $\frac{12}{2}$ methods, provide $1-2 \mu \mathrm{m}$, which is one order of magnitude larger than our data. This remarkable difference in $\lambda_{\|}(0)$ between the experimental methods can be explained in terms of the different "fieldpenetrated portion for evaluating $\lambda$ " as displayed in Fig. 1 The details are discussed later.

In the slow-cooled state, the number of ethylenedisorders will be small and thus it is fruitful to compare with the London penetration depth $\lambda_{\mathrm{L}}(0)$. It can be evaluated by means of the relation: $\lambda_{\mathrm{L}}(0)=$ $(c / e)\left(m^{*} / 4 \pi n_{\mathrm{s}}(0)\right)^{1 / 2}$, where $m^{*}$ is the effective mass and $n_{\mathrm{s}}(0)$ the carrier density. For the $\mathrm{Cu}(\mathrm{NCS})_{2}$ salt, Lang et $a l \stackrel{19}{ }$ have estimated $\lambda_{\mathrm{L}}(0)$ to be $410 \pm 40 \mathrm{~nm}$ taking account of both $\alpha$ - and $\beta$-Fermi surfaces contributing to the superconducting state. The values of $\lambda_{\|}(0)$ obtained in the present experiment is in good agreement with $\lambda_{\mathrm{L}}(0)$. This demonstrates that the $\mathrm{Cu}(\mathrm{NCS})_{2}$ salt is very clean and $l_{\|}$is much long enough to neglect the term " $\xi_{0} / l_{\|}$" in eq. (11), resulting in $\lambda_{\|}(0) \approx \lambda_{\mathrm{L}}(0)$. In the $\mathrm{Cu}\left[\mathrm{N}(\mathrm{CN})_{2}\right] \mathrm{Br}$ salt, one obtains $\lambda_{\mathrm{L}}(0)=360-390 \mathrm{~nm}$ by a similar calculation using $m^{*} / m_{0}=5.4-6.7$ estimated from quantum oscillation investigations $\mathrm{s}^{7.8 .9}$ and $n_{\mathrm{s}}(0)=1.2 \times 10^{21} \mathrm{~cm}^{-3}$ ( $\beta$ orbital). Thus, $\lambda_{\|}(0)$ is much longer than the value of $\lambda_{\mathrm{L}}(0)$ evaluated. This can also be understood in terms of eq. (11); in the $\mathrm{Cu}\left[\mathrm{N}(\mathrm{CN})_{2}\right] \mathrm{Br}$ salt, " $\xi_{0} / l_{\|}$" should not be neglected even in the slow-cooled state because $l_{\|}$is much

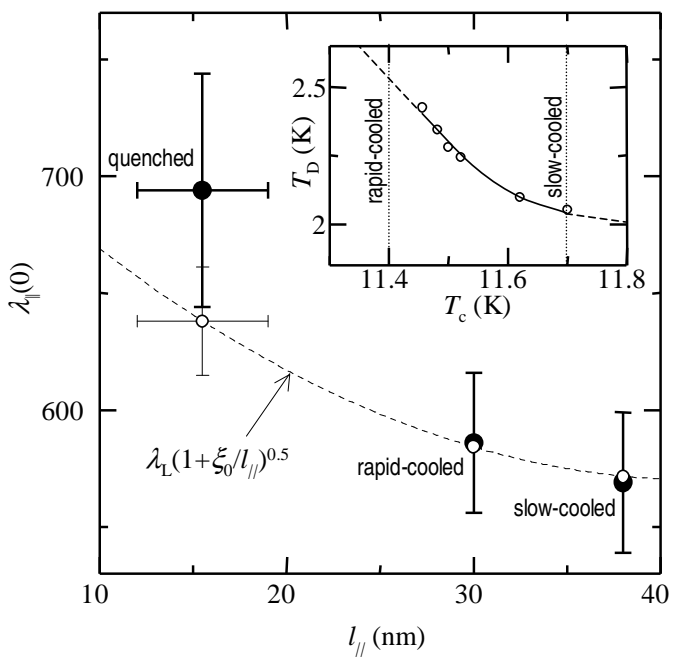

FIG. 8: In-plane penetration depth as a function of the inplane mean free path $l_{\|}$for $\kappa$-(BEDT-TTF $)_{2} \mathrm{Cu}\left[\mathrm{N}(\mathrm{CN})_{2}\right] \mathrm{Br}$ (closed circles). Small open circles are calculated from the London model within the local-clean approximation with a fitting parameter $\lambda_{\mathrm{L}}(0)=523 \mathrm{~nm}$. The broken curve is a guide to the eye. Inset shows the relationship between Dingle temperature $T_{\mathrm{D}}$ and the superconducting transition temperature $T_{\mathrm{c}}$, extracted from the literature. ${ }^{9}$

shorter than that of the $\mathrm{Cu}(\mathrm{NCS})_{2}$ salt. Therefore the effect of $l_{\|}$, i.e., impurity (i.e., cooling-rate as mentioned below) on $\lambda_{\|}$is more effective on the $\mathrm{Cu}\left[\mathrm{N}(\mathrm{CN})_{2}\right] \mathrm{Br}$ salt than the $\mathrm{Cu}(\mathrm{NCS})_{2}$ salt.

Before discussing the cooling-rate effect on $\lambda_{\|}(0)$, we review the impurity effect by ethylene-disorders in the $\mathrm{Cu}\left[\mathrm{N}(\mathrm{CN})_{2}\right] \mathrm{Br}$ salt. It will be believed that the number of the ethylene-disorders increases with cooling faster. Although no direct observation of the disorders has been succeeded in such as X-ray structural studies at low temperatures, 29 the number of the disorders has been proposed to be about 5-20\% $\frac{37.38}{3}$ Moreover, the investigation of the $\mathrm{SdH}$ effect under the control of disorders 9 implies that these disorders can work as an electron (quasiparticle)-scattering origin. They have found a relationship between $T_{\mathrm{c}}$ and the Dingle temperature $T_{\mathrm{D}}$, as shown in the inset of Fig. 8. With decreasing $T_{\mathrm{c}}$, $T_{\mathrm{D}}$ monotonically increases. Generally $T_{\mathrm{D}}$ reflects the sample purity, since the mean free path $l$ is expressed as $l=v_{\mathrm{F}} \hbar / 2 \pi k_{\mathrm{B}} T_{\mathrm{D}}$, where $v_{\mathrm{F}}$ is the Fermi velocity. Therefore, a state with larger $T_{\mathrm{D}}$ corresponds to a more dirty system with shorter $l$. In the following, we explain the increase of $\lambda_{\|}(0)$ by cooling faster in the $\mathrm{Cu}\left[\mathrm{N}(\mathrm{CN})_{2}\right] \mathrm{Br}$ salt on the basis of eq. (II) using the mean free path evaluated.

As shown in the inset of Fig. $8 T_{\mathrm{D}}$ is about $2.0 \mathrm{~K}$ and $2.5 \mathrm{~K}$ for the slow-cooled and rapid-cooled state, respectively. From these values, $l_{\|}$is obtained as $38 \mathrm{~nm}$ (slow-cooled) and $30 \mathrm{~nm}$ (rapid-cooled). In a state with much lower $T_{\mathrm{c}}$, like as being $10.9 \mathrm{~K}$ for the quenched state, the $\mathrm{SdH}$ oscillation amplitudes are considerably 
suppressed, $\stackrel{9}{=}$ and thus $T_{\mathrm{D}}$ has not been obtained. Then an extrapolation to $T_{\mathrm{c}}=10.9 \mathrm{~K}$ for the quenched state gives a very rough value of $T_{\mathrm{D}} \sim 5 \pm 1 \mathrm{~K}$, corresponding to $l_{\|}=12-19 \mathrm{~nm}$. This is still larger than $\xi_{\|}=2.4-3.7$ $\mathrm{nm}^{11.27}$, and therefore the clean-local limiting London model is suitable to apply to the present systems even in the quenched state. Figure 8 shows $\lambda_{\|}(0)$ obtained experimentally as a function of the estimated $l_{\|}$(filled circles). The corresponding values of $\lambda_{\|}(0)$ evaluated from eq. (11) is also plotted using a fitting parameter of $\lambda_{\mathrm{L}}(0)$ $=523 \mathrm{~nm}$ and values of $\xi_{0}$ calculated from the relation $\xi_{0}=a \hbar v_{\mathrm{F}} / k_{\mathrm{B}} T_{\mathrm{c}}$, where $a=0.18$ and $v_{\mathrm{F}}=6.2 \times 10^{6} \mathrm{~cm} / \mathrm{s}$ (open circles). The experimental data are in good quantitative agreement with this model. The deviation in the quenched data may be attributed to the ambiguity in the too roughly estimated value of $l_{\|}$. The good accordance with this model demonstrates that the penetration depth is well described in terms of the clean-local approximation, and the introduction of the disorders provides the impurity effect on the electron (quasiparticle)-scattering, resulting in shorter mean free path.

We here comment on the quite large increase of $\lambda_{\|}(0)$ from the experiments in the shielding states by quenching. From the present investigation (in the mixed state), we obtain a ratio of the penetration depth in the faster cooling state (quenched or rapid-cooled) $\left(\lambda_{\|}^{\mathrm{q}}(0)\right)$ to that in the slow-cooled one $\left(\lambda_{\|}^{\mathrm{s}}(0)\right)$ as $\lambda_{\|}^{\mathrm{q}}(0) / \lambda_{\|}^{\mathrm{s}}(0) \sim 1.03-$ 1.21. Aburto et al . $^{25}$ have reported the similar magnitude of the ratio by means of the magnetization measurements at high magnetic-fields, $\lambda_{\|}^{\mathrm{q}}(0) / \lambda_{\|}^{\mathrm{s}}(0) \sim 1.1$, where $\lambda_{\|}(0)=640 \mathrm{~nm}$ (faster cooling of $20 \mathrm{~K} / \mathrm{min}, T_{\mathrm{c}}$ $=11.6 \mathrm{~K}$ ) and $580 \mathrm{~nm}$ (slower cooling of $1.4 \mathrm{~K} / \mathrm{min}, T_{\mathrm{c}}$ $=11.8 \mathrm{~K}$ ). As discussed above, this change of the absolute values is quantitatively consistent with the London model, corresponding to an increase of about $10-20 \%$ by quenching. In other words, the disorders do not lead to more than 10-20\% increase of $\lambda_{\|}(0)$ at most. In contrast, the measurements in the shielding state not only provide much larger $\lambda_{\|}(0)$ in the slow-cooled state, but also result in a very large increase of $\lambda_{\|}(0)$ by quenching (ac susceptibility 21 ), where the ratio $\lambda_{\|}^{\mathrm{q}}(0) / \lambda_{\|}^{\mathrm{s}}(0)$ is estimated to be about 22-67. This increase of $\lambda_{\|}(0)$ by quenching is two orders of magnitude larger than our results, and also the value expected from the London model for bulk samples. On this discrepancy, we point out that these anomalously large values of $\lambda_{\|}(0)$ observed in the shielding state are attributed to a surface effect of samples. In the experimental techniques which observe shielding volumes at very low magnetic-field below $H_{\mathrm{c} 1}$ (like ac susceptibility), the penetration depth is obtained from the difference between the shielding volume and the sample volume, assuming the magnetic-field is penetrating on the crystalline edge (Fig. 1(a)). On the other hand, in magnetic-field much higher than $H_{\mathrm{c} 1}$, the penetration depth is defined to be a decay length of magneticfield from the center of a vortex core (Fig. [1(b)). They should fundamentally take the same value in an ideal sys-

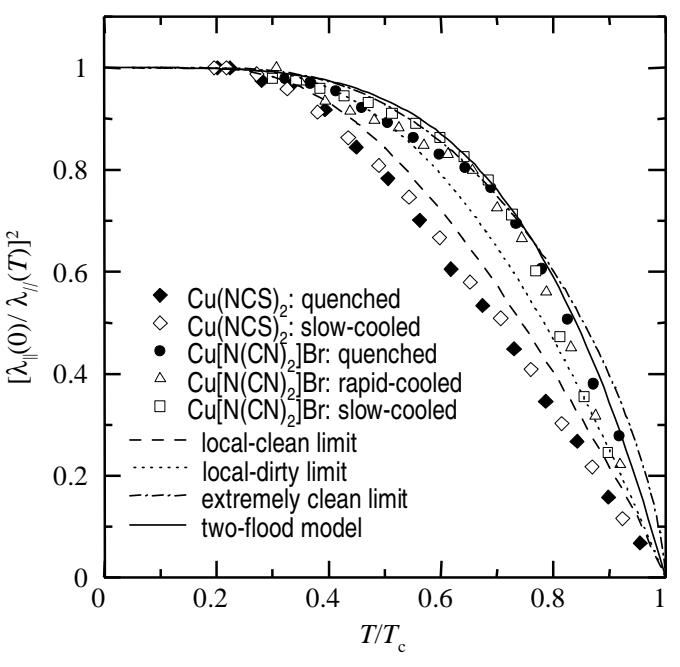

FIG. 9: $\quad\left[\lambda_{\|}(0) / \lambda_{\|}(T)\right]^{2}(\equiv \rho)$ as a function of $T / T_{\mathrm{c}}$. The four curves represent the models based on the conventional BCS theory ${ }^{28}$

tem. But, if a surface state different from the bulk one exists, the penetration depth obtained from low magneticfield technique may deviate from that of the bulk state. As an example, in $\beta$-(BEDT-TTF $)_{2} \mathrm{PF}_{6}$, a structural relaxation in the surface BEDT-TTF molecules has been observed, 39 as a characteristic feature of the surface state. Besides, it is likely that only a small amount of crack or minor damage after quenching leads to a critical influence to the low-field measurements, which may give overestimation of $\lambda_{\|}(0)$. The sample-independence in the data can check this point, but it seems not to be achieved in the literature ${ }^{21}$ This might cause a serious problem on the temperature dependence of the penetration depth as well, and thus a reconsideration will be needed for the experimental results performed at low magnetic-fields, especially after cooling fast. Although the reports on $\lambda_{\|}(T)$ in the shielding state $10,12.13,20,21$ indicate the power-law behavior of $T^{n}$, it is still controversial whether $n=1,2$, or between 1-2. The discussion of the pairing symmetry from the $T$-dependence of $\lambda_{\|}$may not become conclusive unless this problem on the absolute value of $\lambda_{\|}(0)$ is clarified.

Next we discuss the insensitivity in $\lambda_{\|}(0)$ by quenching for the $\mathrm{Cu}(\mathrm{NCS})_{2}$ salt. In this salt, no change of $\lambda_{\|}(0)$ by quenching is observed within the experimental accuracy in contrast to the $\mathrm{Cu}\left[\mathrm{N}(\mathrm{CN})_{2}\right] \mathrm{Br}$ salt. Nevertheless, $T_{\mathrm{c}}$ in the $\mathrm{Cu}(\mathrm{NCS})_{2}$ salt slightly decreases by quenching as well as the $\mathrm{Cu}\left[\mathrm{N}(\mathrm{CN})_{2}\right] \mathrm{Br}$ salt. This implies that the ethylene-disorders are surely introduced to the system after quenching in the $\mathrm{Cu}(\mathrm{NCS})_{2}$ salt as well, and thus the mean free path will be also reduced. In a high-quality sample, the mean free path in the slow-cooled state $l_{\|} \sim$ $200 \mathrm{~nm}$ is obtained from $T_{\mathrm{D}}=0.28 \mathrm{~K} \underline{\underline{\underline{3}}}$ As an example, assuming that $l_{\|}$becomes half of this value by quenching, one can estimate $\lambda_{\|}^{\mathrm{q}}(0) / \lambda_{\|}^{\mathrm{s}}(0)=1.01$ by using eq. 
(11), which is smaller than the experimental error $(= \pm 20$ $\mathrm{nm} / 430 \mathrm{~nm} \sim \pm 5 \%)$. Thus the insensitivity in $\lambda_{\|}(0)$ is certainly reasonable as a very clean system with large $l_{\|}$ compared with the $\mathrm{Cu}\left[\mathrm{N}(\mathrm{CN})_{2}\right] \mathrm{Br}$ salt.

Finally we comment on the temperature dependence of the penetration depth. In our investigation, the coolingrate seems not to change the form of $\lambda_{\|}(T)$ as shown in Fig. [7 Figure[9] shows $\left[\lambda_{\|}(0) / \lambda_{\|}(T)\right]^{2}(\equiv \rho)$ as a function of $T / T_{\mathrm{c}}$. In each salt, $\rho\left(T / T_{\mathrm{c}}\right)$ is likely to be well scaled to the same functional form. This indicates no influence of disorder's impurity on $\rho$ as a function of temperature, suggesting that the pairing symmetry is not altered. Unfortunately, the correct estimate of $\lambda_{\|}(T)$ becomes difficult with lowering temperature in the present investigations, because $H_{\text {irr }}$ narrows the reversible magnetization region (shown in Figs. [5(c), 5(d), 6(c), and 6(d)) and the application of the London model becomes unfavorable. This interrupts the accurate definition of $\lambda_{\|}$at low temperatures. We therefore do not conclude the pairing symmetry of the ground states. Nevertheless, we can point out the difference between the two salts. With increasing $T$, a faster decrease of $\rho\left(T / T_{\mathrm{c}}\right)$ in the $\mathrm{Cu}(\mathrm{NCS})_{2}$ salt is observed than that in the $\mathrm{Cu}\left[\mathrm{N}(\mathrm{CN})_{2}\right] \mathrm{Br}$ salt. In order to compare with the conventional BCS theory, several models ${ }^{28}$ are displayed in Fig. 9] The data of the $\mathrm{Cu}(\mathrm{NCS})_{2}$ salt seem to agree with the local-clean limit model, while those of the $\mathrm{Cu}\left[\mathrm{N}(\mathrm{CN})_{2}\right] \mathrm{Br}$ salt are located in between the two-flood and local-dirty limits. These trends are consistent with the literatures, $\stackrel{19,24}{ }$ which may reflect the difference in the sample purity between the two salts.

In the above discussions, we adopted the conventional
London model, but it does not exclude the unconventional pairing symmetries of these materials. The controversial problem on the functional form of $\lambda_{\|}(T)$ at low temperatures is still an open question.

\section{CONCLUSION}

In conclusion, we report the dc magnetization measurements under the control of cooling-rate for $\kappa$-(BEDT$\mathrm{TTF})_{2} X\left(X=\mathrm{Cu}(\mathrm{NCS})_{2}\right.$ and $\left.\mathrm{Cu}\left[\mathrm{N}(\mathrm{CN})_{2}\right] \mathrm{Br}\right)$. The in-plane penetration depth is evaluated from the slope of the linear region in $M$ vs $\ln H$ plot. We quantitatively explain the behavior of $\lambda_{\|}(0)$ by cooling fast for both salts in terms of the local-clean approximation. In the $\mathrm{Cu}(\mathrm{NCS})_{2}$ salt, the cooling-rate independent $\lambda_{\|}(0)$ $=430 \pm 20 \mathrm{~nm}$ is obtained within the experimental accuracy, while $T_{\mathrm{c}}$ is slightly reduced. This implies that this salt is described as a very clean system. In contrast, a distinct increase of $\lambda_{\|}(0)$ in the $\mathrm{Cu}\left[\mathrm{N}(\mathrm{CN})_{2}\right] \mathrm{Br}$ salt is observed: $570 \pm 30 \mathrm{~nm}$ (slow-cooled), $590 \pm 30 \mathrm{~nm}$ (rapidcooled), and $690 \pm 50 \mathrm{~nm}$ (quenched). This increase of the penetration depth is quantitatively in good agreement with the London model as shown in Fig. 8 This demonstrates that the ethylene-disorders introduced by cooling faster increase the electron (quasiparticle)-scattering, resulting in shorter mean free path.

This research was partly supported by the Ministry of Education, Science, Sports and Culture, Grant-in-Aid for Encouragement of Young Scientists.
1 T. Ishiguro, K. Yamaji and G. Saito: Organic Superconductors, 2nd ed. (Springer, Berlin, 1998).

2 N. Harrison, J. Caulfield, J. Singleton, P. H. P. Reinders, F. Herlach, W. Hayes, M. Kurmoo and P. Day: J. Phys.: Condens. Matter 8 (1996) 5415; eariler references are therein.

3 T. Sasaki, W. Biberacher, K. Neumaier, W. Hehn, K. Andres and T. Fukase: Phys. Rev. B 57 (1998) 10889.

${ }^{4}$ H. Ito, S. M. Hayden, P. J. Meeson, M. Springford and G. Saito: J. Supercond. 12 (1999) 525.

${ }^{5}$ N. J. Clayton, H. Ito, S. M. Hayden, P. J. Meeson, M. Springford and G. Saito: Phys. Rev. B 65 (2002) 064515.

6 J. Singleton, C. H. Mielke, W. Hayes and J. A. Schlueter: J. Phys.: Condens. Matter 15 (2003) L203.

7 C. H. Mielke, N. Harrison, D. G. Rickel, A. H. Lacerda, R. M. Vestal and L. K. Montgomery: Phys. Rev. B 56 (1997) R4309.

${ }^{8}$ H. Weiss, M. V. Kartsovnik, W. Biberacher, E. Steep, E. Balthes, A. G. M. Jansen, K. Andres and N. D. Kushch: Phys. Rev. B 59 (1999) 12370.

9 T. F. Stalcup, J. S. Brooks and R. C. Haddon: Phys. Rev. B 60 (1999) 9309.

10 D. Achkir, M. Poirier, C. Bourbonnais, G. Quirion, C. Lenoir, P. Batail and D. Jérome: Phys. Rev. B 47 (1993) 11595.
11 M. Dressel, O. Klein, G. Grüner, K. D. Carlson, H. H. Wang and J. M. Williams: Phys. Rev. B 50 (1994) 13603.

12 A. Carrington, I. J. Bonalde, R. Prozorov, R. W. Giannetta, A. M. Kini, J. Schlueter, H. H. Wang, U. Geiser and J. M. Williams: Phys. Rev. Lett. 83 (1999) 4172.

13 K. Kanoda, K. Akiba, K.Suzuki, T. Takahashi and G. Saito: Phys. Rev. Lett. 65 (1990) 1271.

14 P. A. Mansky, P. M. Chaikin and R. C. Haddon: Phys. Rev. B 50 (1994) 15929.

15 D. R. Harshman, R. N. Kleiman, R. C. Haddon, S. V. C-Hicks, M. L. Kaplan, L. W. Rupp, Jr., T. Pfiz, D. Ll. Williams and D. B. Mitzi: Phys. Rev. Lett. 64 (1990) 1293.

16 D. R. Harshman, A. T. Fiory, R. C. Haddon, M. L. Kaplan, T. Pfiz, E. Koster, I. Shinkoda and D. Ll. Williams: Phys. Rev. B 49 (1994) 12990.

17 S. L. Lee, F. L. Pratt, S. J. Blundell, C. M. Aegerter, P. A. Pattenden, K. H. Chow, E. M. Forgan, T. Sasaki, W. Hayes and H. Keller: Phys. Rev. Lett. 79 (1997) 1563.

18 L. Ya. Vinnikov, T. L. Barkov, M. V. Kartsovnik and N. D. Kushch: Phys. Rev. B 61 (2000) 14358.

19 M. Lang, N. Toyota, T. Sasaki and H. Sato: Phys. Rev. Lett. 69 (1992) 1443.

20 M. Pinterić, S. Tomić, M. Prester, D. Drobac, O. Milat, K. Maki, D. Schweitzer, I. Heinen and W. Strunz: Phys. 
Rev. B 61 (2000) 7033.

21 M. Pinterić, S. Tomić, M. Prester, D. Drobac and K. Maki: Phys. Rev. B 66 (2002) 174521.

${ }^{22}$ L. P. Le, G. M. Luke, B. J. Sternlieb, W. D. Wu, Y. J. Uemura, J. H. Brewer, T. M. Riseman, C. E. Stronach, G. Saito, H. Yamochi, H. H. Wang, A. M. Kini, K. D. Carlson and J. M. Williams: Phys. Rev. Lett 68 (1992) 1923.

${ }^{23}$ F. L. Barkov, L. Ya. Vinnikov, M. V. Kartsovnik and N. D. Kushch: Physica C 385 (2003) 568.

24 M. Lang, N. Toyota, T. Sasaki and H. Sato: Phys. Rev. B 46 (1992) 5822.

25 A. Aburto, L. Fruchter and C. Pasquier: Physica C 303 (1998) 185.

26 The in-plane mean free path $l_{\|}$is estimated from the Dingle temperature and effective mass obtained from the quantum oscillation measurements in Refs. 23456 . In contrast, $l_{\|}$ obtained from the in-plane residual resistivity ${ }^{11.16}$ is much shorter than these values: $15-30 \mathrm{~nm}$. This discrepancy may be related to the domain structure as discussed in Ref. 6 .

27 H. Ito, M. Watanabe, Y. Nogami, T. Ishiguro, T. Komatsu, G. Saito and N. Hosoito: J. Phys. Soc. Jpn. 60 (1991) 3230.

${ }^{28}$ M. Tinkham: Introduction to Superconductivity, 2nd ed. (McGraw-Hill, New York, 1996).

29 U. Geiser, A. J. Schultz, H. H. Wang, D. M. Watkins, D.
L. Stupka, J. M. Williams, J. E. Schirber, D. L. Overmyer, D. Jung, J. J. Novoa and M. -H. Whangboo: Physica C 174 (1991) 475.

${ }^{30}$ X. Su, F. Zuo, J. A. Schlueter, M. E. Kelly and J. M. Williams: Phys. Rev. B 57 (1998) R14056.

31 N. Yoneyama, A. Higashihara, T. Sasaki, T. Nojima and N. Kobayashi: J. Phys. IV (France) (2004) in press.

32 P. G. de Gennes: Superconductivity of Metals and Alloys (Benjamin, New York, 1966).

33 Z. Hao and J. R. Clem: Phys. Rev. Lett. 67 (1991) 2371.

34 M. Tokumoto, N. Kinoshita, Y. Tanaka, T. Kinoshita and H. Anzai: Synth. Met. 103 (1999) 1971.

35 H. Taniguchi, A. Kawamoto and K. Kanoda: Phys. Rev. B 59 (1999) 8424.

36 N. Yoneyama, T. Sasaki, T. Nishizaki and N. Kobayashi: J. Phys. Soc. Jpn. 73 (2004) in press (cond-mat/0108532).

37 H. Akutsu, K. Saito and M. Sorai: Phys. Rev. B 61 (2000) 4346.

38 J. Müller, M. Lang, F. Steglich, J. A. Schlueter, A. M. Kini and T. Sasaki: Phys. Rev. B 65 (2002) 144521.

39 M. Ishida, O. Takeuchi, T. Mori and H. Shigekawa: Phys. Rev. B 64 (2001) 153405. 